\title{
OPEN Synthesizing environmentally friendly non-silicone oxygen bleaching stabilizer for linen yarn using oligomeric acrylic acid
}

Jie Liuํ \& Chun Lv²凶

Using potassium peroxodisulfate as an initiator and acrylic acid as a monomer, an acrylic acid oligomer was synthesized and then compounded with magnesium salt to form a non-silicone oxygen bleaching stabilizer. By investigating the effects of reaction temperature, reaction time, initiator concentration, monomer concentration, and magnesium salt dosage on product performance, the effect of stabilizers on linen yarn bleaching was analyzed. The synthetic conditions of oxygen bleaching stabilizer were determined by orthogonal test method, namely, acrylic acid monomer concentration $25 \%$, initiator dosage $5 \%$, oligomeric acrylic acid and magnesium salt compound ratio $5: 1$, reaction temperature $65^{\circ} \mathrm{C}$, reaction time $4 \mathrm{~h}$. At this time, the chelated iron value of the product was as high as $239.314 \mathrm{mg} / \mathrm{g}$, and the chelated calcium value also reached $145.000 \mathrm{mg} / \mathrm{g}$. The dosage of the synthesized stabilizer were determined to be $4 \mathrm{~g} / \mathrm{L}$ through indicators such as the decomposition rate of hydrogen peroxide and whiteness. The results showed that the environmentally friendly non-silicone oxygen bleaching stabilizer not only had a good ability to inhibit the decomposition of hydrogen peroxide, but also provided bleached linen yarn with a superior degree of whiteness and less metal ion residue, which can effectively solve the "silicon scale" problem and improve the quality of the pre-treatmented products.

Oxygen bleaching stabilizer (OBS) is an essential auxiliary in hydrogen peroxide $\left(\mathrm{H}_{2} \mathrm{O}_{2}\right)$ bleaching. Hydrogen peroxide bleaching is usually carried out under alkaline conditions, and the trace metals and ions contained in the bleaching solution can catalyze the decomposition of hydrogen peroxide ${ }^{1-3}$. For example, $\mathrm{Fe}^{3+}$ can catalyze the ineffective decomposition of hydrogen peroxide, and the hydroxyl radicals generated can attack and destroy the most stable $\mathrm{C}-\mathrm{H}$ bond when highly activated, causing the fiber brittle. Therefore, oxygen bleaching stabilizer must be applied during hydrogen peroxide bleaching to slow down the rapid decomposition of hydrogen peroxide and protect the fiber from damage ${ }^{4-6}$.

Oxygen bleaching stabilizers mainly include inorganic stabilizers and organic stabilizers. According to its chemical composition, it can be divided into two types: silicon-containing stabilizer and non-silicon-containing stabilizer; According to its component, there are single component and compound type. According to its stability mechanism, there are two kinds: adsorption type and chelating type oxygen bleaching stabilizer ${ }^{7-9}$. The most primary types of oxygen bleaching stabilizers are sodium silicate and phosphonates. Sodium silicate, as an oxygen bleaching stabilizer commonly used in the traditional process, belongs to inorganic adsorption products containing silicon. It has been used in the bleaching process of various textile materials because of its good bleaching effect and low cost. However, it will easily generate insoluble salt with metal ions such as $\mathrm{Ca}^{2+}$ and $\mathrm{Mg}^{2+} \mathrm{during}$ the production process, that is silica scale. The silica scale deposited on the bleaching equipment will bring difficulties to the cleaning work of the equipment. At the same time, the silica scale adhered on the product is easy to cause the fabric rough handle, dyeing unevenly, and even produce white spots and other defects ${ }^{10-12}$. Therefore, the development and application of non-silicon oxygen bleaching stabilizer is increasingly important.

Non-silicon oxygen bleaching stabilizers mainly include phosphonates and macromolecule compounds. Phosphine amino acid, methylene phosphonic acid and ethylenediamine (acid) has been studied most in the past. Christiansen filed a patent for a method for the application of diethylenetriamine pentamethyl phosphonic acid (DTPMPA) in combination with sodium polyacrylate or hydroxyl acrylate polymer as an oxygen bleaching stabilizer ${ }^{13-15}$. Rong-qi Chen ${ }^{16,17}$ developed a phosphonic acid ester type oxygen bleaching stabilizer, which was 
an excellent stabilizer with both adsorption and chelation functions. Its molecular structure contained oligomers with spatial coordination groups such as phosphoric acid group and hydroxyl group, which was resistant to concentrated alkali, highly concentrated hydrogen peroxide and high temperature. These phosphonates OBS caused water quality deterioration, so not to be recommended to continue development.

Macromolecule stabilizers meet the ecological standardS. Zhu Wenjun et al. ${ }^{18}$ synthesized a polycarboxylic acid with maleic anhydride as the main raw material, and mixed it with an appropriate amount of magnesium chloride to produce OBS capable of both adsorption and chelation. Zhou Lizheng and Luo Kunping ${ }^{19}$ developed a highly efficient non-silicon OBS with strong complexation and adsorption on metal ions. The bleached fabrics felt soft and had good anti-scale effect. Qi Wenyu and Huang Wenbo ${ }^{20}$ developed the OBS FK-601 by polymerizing acrylamide as monomer and then compounding, which eradicated the defects of silica scale adhesion, effectively inhibited the decomposition of hydrogen peroxide and avoided the damage to the fiber. Water-soluble graft polymer including the monomers such as acrylamide, sodium acrylate, (3,4-dihydroxy phenyl) methane sulfonate, $\mathrm{N}$-(trihydroxymethyl) methyl acrylamide, (2-hydroxy phenyl) methane sulfonate has been used as OBS, but the effect applied alone was not better than that in combination with EDTA, DTPA, sodium gluconate, citric acid and quaternary ammonium salt ${ }^{21-23}$. The maleic acid and styrene copolymer, maleic acid and propylene copolymer, polyhydroxycarboxylic acid were used as OBS. There are also modified chitosan synthesis N-(3,5-Diethylamino benzophthalide) chitosan, $\mathrm{N}$-(4-ethylamino benzophthalide) chitosan polymercontaining aromatic amines that can chelate metals, but the cost is very high ${ }^{24,25}$.

In this paper, a type of non-silicon oxygen bleaching stabilizer using acrylic acid and magnesium sulfate as the main raw material was synthesized and applied to the linen yarn. Acrylic acid polymerizes under the action of potassium peroxodisulfate as the initiator to generate oligoacrylic acid, which had good chelating ability and can effectively chelate various metal ions. After compounding with magnesium salt, magnesium hydroxide colloid was formed in the neutral medium, which had strong adsorption capacity. Therefore, the magnesium oligomer acrylate stabilizer can effectively inhibit the catalysis of metal ions, thereby inhibiting the ineffective decomposition of hydrogen peroxide ${ }^{26}$. Experiment results proved that the non-silicon OBS had little damage on textiles materails, and the most important advantage was that the non-silicon OBS has no pollution to the environment and no affinity with fiber, easy to wash out after bleaching, as well as no influences to the bleached products. In addition, this non-silicon OBS is also without phosphorus, meets the environmental requirements. Therefore, synthesis and applications of the synthesized non-silicon oxygen bleaching stabilizer had very vital significance in the bleaching process. It can replace sodium silicate in the pretreatment process and solve the problem of silica scale in the bleaching process.

\section{Methods}

Materials. The linen yarn used in this study was kindly provided by Qiqihar Jinya Group (China).

Chemicals. Acrylic acid (chemically pure) as a monomer and potassium peroxodisulfate as an initiator were perchased from Tianjin cameo chemical reagent Co. Ltd. (China). Hydrogen peroxide $(30 \% \mathrm{w} / \mathrm{w})$ as a bleaching agent, magnesium sulfate, ammonium iron (III) sulfate, sodium hydroxide, potassium permanganate, ammonium chloride, ammonia, sulfuric acid, calcium acetate and sodium oxalate were perchased from Tianjin kaitong chemical reagent Co. Ltd. (China). Unless otherwise specified, all the other chemicals used in this study were reagent-grade chemicals.

Synthesis of non-silicon OBS. This stabilizer was synthesized with solution polymerization. A certain amount of the initiator potassium perdisulfite $(2,3,4,5,6 \%$ by the mass of acrylic monomer) and distilled water were added to a three-necked flask containing an electric stirrer, thermometer and reflux condensing unit. Stirring and heating until the initiator potassium perdisulfite was completely dissolved, acrylic acid monomer (10, $15,20,25,30 \%)$ and distilled water were added into the initiator solution. Reacted at $\left(50,55,60,65,70{ }^{\circ} \mathrm{C}\right)$ for $(2$, $3,4,5) \mathrm{h}$, the light yellow viscous oligoacrylic acid was obtained. Finally added $(1,2,3,4,5 \mathrm{~g}$ based on $10 \mathrm{~g}$ acrylic acid) magnesium sulfate, continue to stirring for $1 \mathrm{~h}$, then stop the reaction, and non-silicon OBS was obtained.

Determination of chelated iron value. The stabilizer solution was titrated with a standard $\mathrm{Fe}^{3+}$ solution. The titration end point was when the stabilizer solution appeared turbidity or color change. The ability to chelated iron ions was expressed in milligrams of chelated trivalent iron ions per gram of the stabilizer. Chelated iron value was calculated by Eq. (1).

$$
\text { Chelated iron value }=V /(G \times 10 / 100)
$$

where $\mathrm{V}$ is the volume of $1.0 \mathrm{~g} / \mathrm{L}$ standard $\mathrm{Fe}^{3+}$ solution consumed $(\mathrm{ml}), \mathrm{G}$ is the weight of the stabilizer used $(\mathrm{g})$.

Determination of chelated calcium value. The stabilizer solution was titrated with calcium standard solution, using sodium oxalate as an indicator. The titration end point was when the stabilizer solution appeared white calcium oxalate precipitation. Chelated calcium value was calculated by Eq. (2).

$$
\text { Chelated calcium value }=(V \times C \times 100) /(G \times 25 / 100)
$$

where $\mathrm{V}$ is the volume of the calcium acetate solution consumed $(\mathrm{ml}), \mathrm{C}$ is the concentration of the calcium acetate solution $(\mathrm{mol} / \mathrm{L}), \mathrm{G}$ is the weight of the stabilizer used $(\mathrm{g})$. 


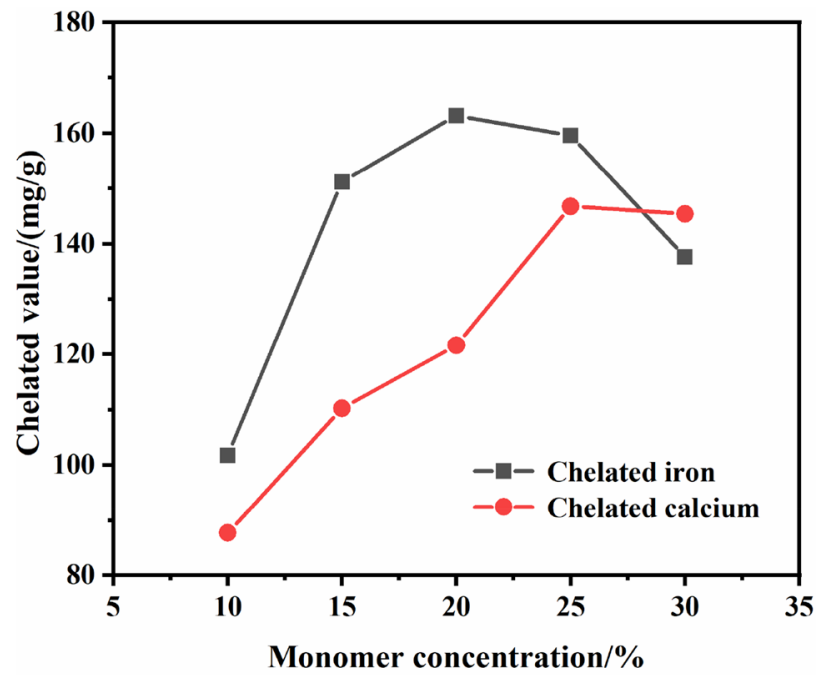

Figure 1. Effect of acrylic monomer concentration on chelated value.

Determination of decomposition rate of hydrogen peroxide. The decomposition rate (DR) of $\mathrm{H}_{2} \mathrm{O}_{2}$ was determined with the stanard solution of potassium permanganate and calculated by Eq. (3).

$$
D R=\left(V_{0}-V_{1}\right) / V_{0} \times 100 \%
$$

where $\mathrm{V}_{0}$ is the volume of the standard solution of potassium permanganate consumed by $5 \mathrm{ml}$ of bleaching bath before bleaching, $\mathrm{V}_{1}$ is the volume of the standard solution of potassium permanganate consumed by $5 \mathrm{ml}$ of bleaching bath after bleaching ${ }^{27-29}$.

Whiteness measurement. Refer to Textile Fiber Whiteness Chroma Test Method GB/T 17644-2008 for determination. The linen roving yarns was measured on a spectrophotometer (YQ-Z-48A whiteness color tester, China) using a $\mathrm{D}_{65}$ illuminant and $10^{\circ}$ standard observer. Each sample was about $80 \mathrm{~mm}$ long and was closely arranged on the pallet of the whiteness color tester. Each sample was rotated in four different directions for measurement. An average of four readings was calculated for each sample.

\section{Results and discussion}

Synthesis of non-silicon oxygen bleaching stabilizer. Effect of acrylic monomer concentration on chelated value. In this experiment, water was selected as the solvent, the monomer was $10 \mathrm{~g}$ acrylic acid, the amount of distilled wate was added to change the acrylic monomer concentration upto 10, 15, 20, 25, 30\%, respectively. Potassium persulfate dosage was $4 \%(2.5 \mathrm{~g})$, reacted at $70{ }^{\circ} \mathrm{C}$ for $4 \mathrm{~h}$, then added $2 \mathrm{~g}$ magnesium sulfate, continued to react for $1 \mathrm{~h}$. The effect of acrylic monomer concentration on chelated value is shown in Fig. 1.

It can be seen from Fig. 1, the chelated value increased gradually with increasing of monomer concentration at the beginning. The reason is that with increasing of monomer concentration, the polymerization rate accelerated, the monomer conversion rate improved, and polymers amount increased, that is, the chelating ability was gradually enhanced. The monomer concentration increased to $20 \%$, the chelated iron value reached its maximum level, and increased to $25 \%$, the chelated calcium value reached its maximum, but the chelated iron value decreased. After that, the chelated iron value and chelated calcium valuehe decreased with the monomer concentration increased. It indicates that appropriate acrylic acid used was beneficial to the chelating effectiveness. When acrylic acid monomer added too much, the molecular weight of the polymerized products increased, the viscosity of the products also increased and leaded to operate difficultly, thus the chelating effect decreased. In view of the reason that $\mathrm{Fe}^{3+}$ had stronger effect on accelerating the decomposition of $\mathrm{H}_{2} \mathrm{O}_{2}$, the concentration of acrylic acid should be about $20 \%$.

Effect of magnesium sulfate dosage on chelated value. The concentration of acrylic monomer was $20 \%$ (10 g acrylic acid and $40 \mathrm{~g}$ distilled water), potassium persulfate was $4 \%$ of the mass of acrylic acid used, reacted at $70{ }^{\circ} \mathrm{C}$ for $4 \mathrm{~h}$, then added $1,2,3,4,5 \mathrm{~g}$ magnesium sulfate respectively, continued to react for $1 \mathrm{~h}$. The effect of magnesium sulfate dosage on chelated value is shown in Fig. 2.

It can be seen from Fig. 2, the magnesium sulfate dosage was between 1 and $2 \mathrm{~g}$, the chelated iron value increased from about $90 \mathrm{mg} / \mathrm{g}$ to about $160 \mathrm{mg} / \mathrm{g}$. The reason is that magnesium salt existed in the form of magnesium hydroxide micelles in the alkaline medium, while magnesium salt dosage increased, the number of generated micelles increased and enhanced the adsorption capacity. After that, the chelated iron value and chelated calcium valuehe decreased with the magnesium sulfate dosage increasing. The reason is that excessive magnesium salt leaded to an increase in the number of magnesium hydroxide micelles with positive charges, and the carboxyl groups with negative charges on the polymer chain were adsorbed and chelated, thus reduced the 


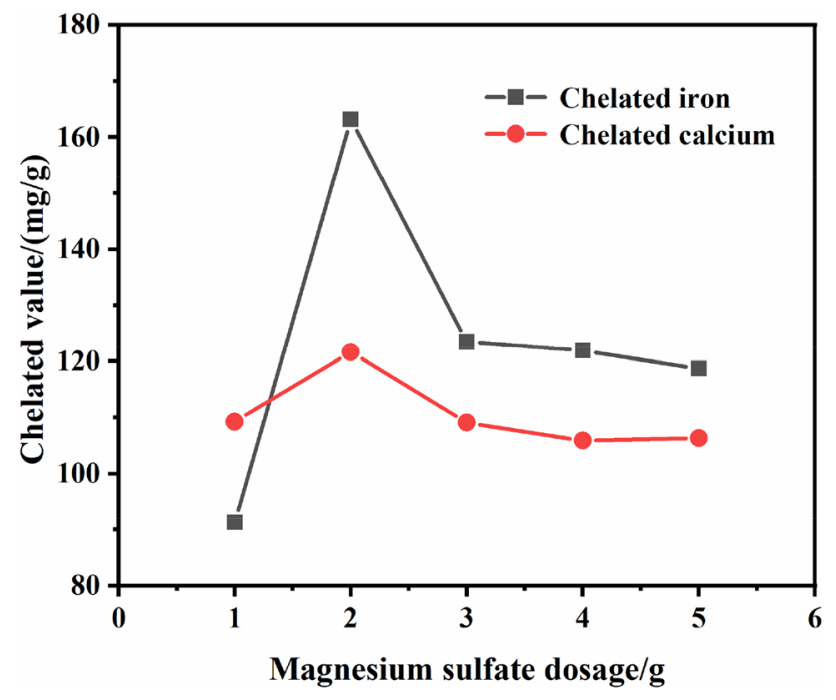

Figure 2. Effect of magnesium sulfate dosage on chelated value.

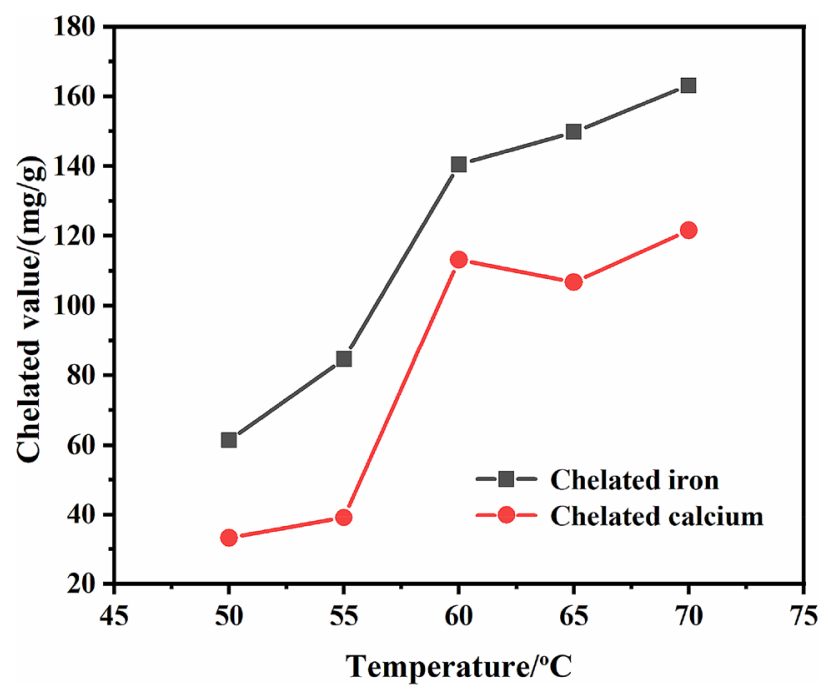

Figure 3. Effect of temperature on chelated value.

number of effective carboxyl negative ions and affected the chelation of positive metal ions by carboxyl negative ions $\mathrm{s}^{30,31}$. Although the change of chelated calcium value was not as obvious as that of chelated iron value, it also reflected the same trend. The optimal magnesium sulfate dosage should be $2 \mathrm{~g}$, that is, the mass ratio of acrylic acid to magnesium sulfate should be $5: 1$.

Effect of temperature on chelated value. The acrylic monomer concentration was $20 \%$, potassium persulfate dosage was $4 \%$, respectively reacted at $50,55,60,65,70^{\circ} \mathrm{C}$ for $4 \mathrm{~h}$, then added $2 \mathrm{~g}$ magnesium sulfate, continued to react for $1 \mathrm{~h}$. The effect of temperature on chelated value is shown in Fig. 3.

It can be seen from Fig. 3, the chelated value increased gradually with temperature rised. With increasing of the polymerized temperature, the movement of each molecule in the reacting system was accelerated, and the collision probability between molecules increased. The polymer viscosity became larger and larger, indicating that the molecular weight of the polymer also increased. The chelating ability was improved by the formation of more polymers ${ }^{32}$. When the temperature continued to rise to $75^{\circ} \mathrm{C}$, the reactants was too viscous to obtain the final product. So the temperature of synthetic reaction should be at about $70{ }^{\circ} \mathrm{C}$.

Effect of initiator dosage on chelated value. The concentration of acrylic monomer was $20 \%$, the dosage of potassium persulfate as initiator was $2,3,4,5,6 \%$ by the mass of acrylic monomer, respectively, reacted at $70{ }^{\circ} \mathrm{C}$ 


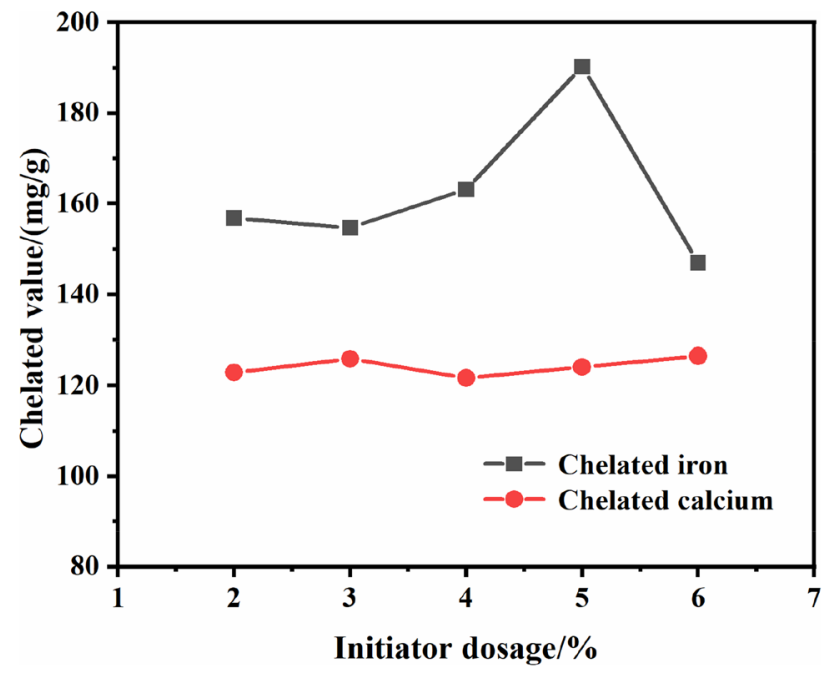

Figure 4. Effect of initiator dosage on chelated value.

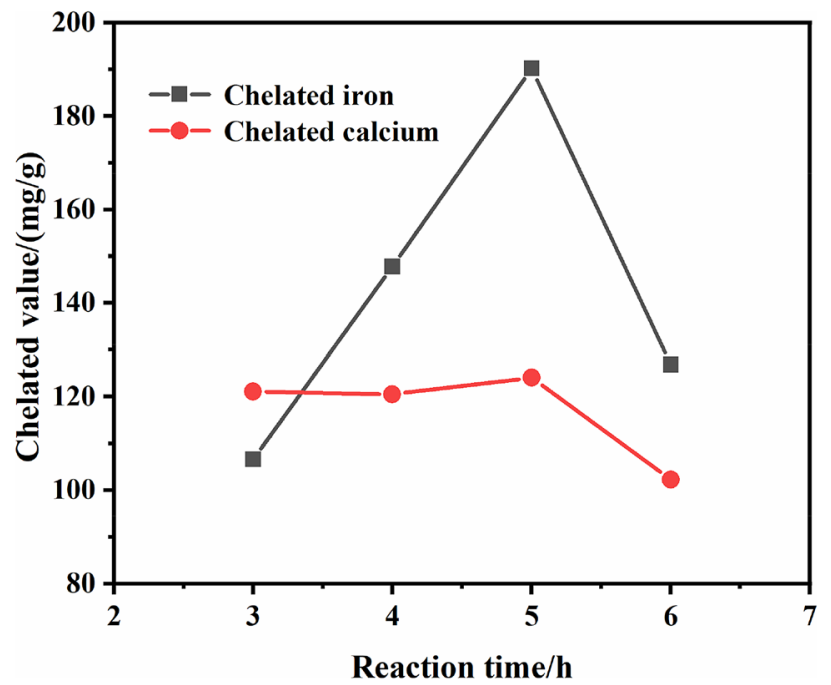

Figure 5. Effect of reaction time on chelated value.

for $4 \mathrm{~h}$, then added $2 \mathrm{~g}$ magnesium sulfate, continued to react for $1 \mathrm{~h}$. The effect of initiator dosage on chelated value is shown in Fig. 4.

It can be seen from Fig. 4, when the potassium persulfate dosage increased from 2 to $4 \%$, the chelated value changed gently. The reason is that the initiator dosage was too little to generate a lot of free radicals, the chain initiation efficiency was lower, the monomer conversion rate was also lower, and the effective chelate components were few. When potassium persulfate increased to $5 \%$ of the mass of acrylic acid, the chelated iron value reached the maximum, it indicated that the amount of initiator met the requirements of the polymerization, and the number of free radicals generated was enough to initiate the monomer polymerization. The potassium persulfate dosage increased to $6 \%$, the chelated iron value decreased significantly, indicated that excessive initiator increased the crosslinking degree of monomers, decreased the sensitivity and reduced the chelating performance ${ }^{33}$. The chelated calcium value hardly fluctuated, indicating that initiator dosage had little effect on chelated calcium value. Therefore, the most suitable dosage of potassium persulfate should be $5 \%$ of the mass of acrylic monomer.

Effect of reaction time on chelated value. The concentration of acrylic monomer was $20 \%$, potassium persulfate dosage was $4 \%$, reacted at $70{ }^{\circ} \mathrm{C}$ for $2,3,4,5 \mathrm{~h}$, respectively, then added $2 \mathrm{~g}$ magnesium sulfate, continued to react for $1 \mathrm{~h}$, so the total reaction time was $3,4,5,6 \mathrm{~h}$. The effect of reaction time on chelated value is shown in Fig. 5.

It can be seen from Fig. 5 , in the range of 3-5 h, the chelated iron value showed an upward trend and increased greatly, reached the maximum value at $5 \mathrm{~h}$, while the chelated calcium value almost did not fluctuate in this range. However, when the total reaction time increased to $6 \mathrm{~h}$, the chelated iron value and chelated calcium value decreased significantly, so the optimal reaction time should be $5 \mathrm{~h}$. The reason is that the reaction time was too 


\begin{tabular}{|l|l|l|l|l|l|l|l|}
\hline No & $\boldsymbol{A}\left(\mathbf{M g S O}_{4}\right) / \mathbf{g}$ & $\boldsymbol{B}$ (Acrylic Con.)/\% & $\boldsymbol{C}($ Initiator)/\% & $\begin{array}{l}\boldsymbol{D} \text { (Temperature) } \\
{ }^{\circ} \mathrm{C}\end{array}$ & $\boldsymbol{E}($ Time $) / \mathbf{h}$ & $\begin{array}{l}\text { Chelated iron } \\
\text { value/(mg/g) }\end{array}$ & $\begin{array}{l}\text { Chelated } \\
\text { calcium value/ } \\
(\mathbf{m g} / \mathbf{g})\end{array}$ \\
\hline 1 & $1(1)$ & $10(1)$ & $4.0(1)$ & $60(1)$ & $4.0(1)$ & 90.071 & 32.610 \\
\hline 2 & $1(1)$ & $15(2)$ & $4.5(2)$ & $65(2)$ & $4.5(2)$ & 116.285 & 100.312 \\
\hline 3 & $1(1)$ & $20(3)$ & $5.0(3)$ & $70(3)$ & $5.0(3)$ & 130.273 & 104.441 \\
\hline 4 & $1(1)$ & $25(4)$ & $5.5(4)$ & $75(4)$ & $5.5(4)$ & 143.569 & 140.896 \\
\hline 5 & $1.5(2)$ & $10(1)$ & $4.5(2)$ & $70(3)$ & $5.5(4)$ & 75.859 & 62.396 \\
\hline 6 & $1.5(2)$ & $15(2)$ & $4.0(1)$ & $75(4)$ & $5.0(3)$ & 99.855 & 94.762 \\
\hline 7 & $1.5(2)$ & $20(3)$ & $5.5(4)$ & $60(1)$ & $4.5(2)$ & 136.319 & 119.505 \\
\hline 8 & $1.5(2)$ & $25(4)$ & $5.0(3)$ & $65(2)$ & $4.0(1)$ & 193.126 & 138.606 \\
\hline 9 & $2.0(3)$ & $10(1)$ & $5.0(3)$ & $75(4)$ & $4.5(2)$ & 119.781 & 89.264 \\
\hline 10 & $2.0(3)$ & $15(2)$ & $5.5(4)$ & $70(3)$ & $4.0(1)$ & 124.008 & 98.959 \\
\hline 11 & $2.0(3)$ & $20(3)$ & $4.0(1)$ & $65(2)$ & $5.5(4)$ & 168.725 & 128.614 \\
\hline 12 & $2.0(3)$ & $25(4)$ & $4.5(2)$ & $60(1)$ & $5.0(3)$ & 166.505 & 137.087 \\
\hline 13 & $2.5(4)$ & $10(1)$ & $5.5(4)$ & $65(2)$ & $5.0(3)$ & 136.072 & 76.110 \\
\hline 14 & $2.5(4)$ & $15(2)$ & $5.0(3)$ & $60(1)$ & $5.5(4)$ & 101.748 & 91.174 \\
\hline 15 & $2.5(4)$ & $20(3)$ & $4.5(2)$ & $75(4)$ & $4.0(1)$ & 158.185 & 127.156 \\
\hline 16 & $2.5(4)$ & $25(4)$ & $4.0(1)$ & $70(3)$ & $4.5(2)$ & 176.589 & 145.103 \\
\hline
\end{tabular}

Table 1. The orthogonal Layout.

\begin{tabular}{|c|c|c|c|c|c|c|}
\hline & A & B & C & D & $\mathbf{E}$ & \\
\hline \multirow{9}{*}{ Chelated iron value } & $\mathrm{K}_{1}$ & 480.198 & 421.783 & 535.240 & 494.643 & 565.390 \\
\hline & $\mathrm{K}_{2}$ & 505.159 & 441.896 & 516.834 & 614.208 & 548.974 \\
\hline & $\mathrm{K}_{3}$ & 579.019 & 593.502 & 544.928 & 506.729 & 506.873 \\
\hline & $\mathrm{K}_{4}$ & 572.594 & 679.798 & 539.968 & 521.390 & 489.901 \\
\hline & $\mathrm{k}_{1}$ & 120.0495 & 105.4458 & 133.8100 & 123.6608 & 141.3475 \\
\hline & $\mathrm{k}_{2}$ & 126.2898 & 110.4740 & 129.2085 & 153.5520 & 137.2435 \\
\hline & $\mathrm{k}_{3}$ & 144.7548 & 148.3755 & 136.2320 & 126.6823 & 126.7183 \\
\hline & $\mathrm{k}_{4}$ & 143.1485 & 169.9473 & 134.9920 & 130.3475 & 122.4753 \\
\hline & $\mathrm{R}$ & 24.7053 & 64.5015 & 7.0235 & 29.8912 & 18.8722 \\
\hline \multirow{9}{*}{ Chelated calcium value } & $\mathrm{K}_{1}$ & 378.259 & 260.380 & 401.089 & 380.376 & 397.331 \\
\hline & $\mathrm{K}_{2}$ & 415.269 & 385.207 & 426.951 & 443.642 & 454.184 \\
\hline & $\mathrm{K}_{3}$ & 453.924 & 479.716 & 423.485 & 410.899 & 412.400 \\
\hline & $\mathrm{K}_{4}$ & 439.543 & 561.692 & 435.470 & 452.078 & 423.080 \\
\hline & $\mathrm{k}_{1}$ & 94.5648 & 65.0950 & 100.2723 & 95.0940 & 99.3328 \\
\hline & $\mathrm{k}_{2}$ & 103.8173 & 96.3018 & 106.7378 & 110.9105 & 113.5460 \\
\hline & $\mathrm{k}_{3}$ & 113.4810 & 119.9290 & 105.8713 & 102.7248 & 103.1000 \\
\hline & $\mathrm{k}_{4}$ & 109.8858 & 140.4230 & 108.8675 & 113.0195 & 105.7700 \\
\hline & $\mathrm{R}$ & 18.9162 & 75.3280 & 8.5952 & 17.9255 & 14.2132 \\
\hline
\end{tabular}

Table 2. Analysis of orthogonal experimental results.

short to polymerize completely, and leaded to uneven distribution of molecular weight of the polymerization products, affecting the performance of the final products. On the contrary, the reaction time was too long, the polymerized molecular chain segments continued to polymerize, resulting in a burst polymerization, which increased the molecular weight of the product and also affected its chelating performance.

Orthogonal optimization of synthesis process of non-silicon OBS. Based on the influences of single factor on the chelated effect of the stabilizer, the optimized synthesis process of non-silicon OBS was determined by orthogonal experiment of $\mathrm{L}_{16}\left(4^{5}\right)$. The result is shown in Table 1 , and the range analysis is shown in Table 2 .

It can be seen from Tables 1 and 2, for the chelated iron value, the order of primary and secondary factors was $\mathrm{B}>\mathrm{D}>\mathrm{A}>\mathrm{E}>\mathrm{C}$, the optimization scheme was $\mathrm{A}_{3} \mathrm{~B}_{4} \mathrm{C}_{3} \mathrm{D}_{2} \mathrm{E}_{1}$. For the chelated calcium value, the order was $B>A>D>E>C$, the optimization scheme was $A_{3} B_{4} C_{4} D_{4} E_{2}$. Factor $B$ was the first major factor, for two indicators of chelated iron value and chelated calcium value, the optimal level was $\mathrm{B}_{4}$, so $\mathrm{B}_{4}$ was selected. Factor A was the third main factor for chelated calcium value and was the second main factor for chelated iron value, the optimal level was $A_{3}$, so $A_{3}$ was selected. Factor $D$ was the second main factor for chelated iron value, $D_{2}$ was the best; but for chelated calcium value, $\mathrm{D}_{4}$ was the best. In view of the difficulty of polymerization at $75^{\circ} \mathrm{C}$ 


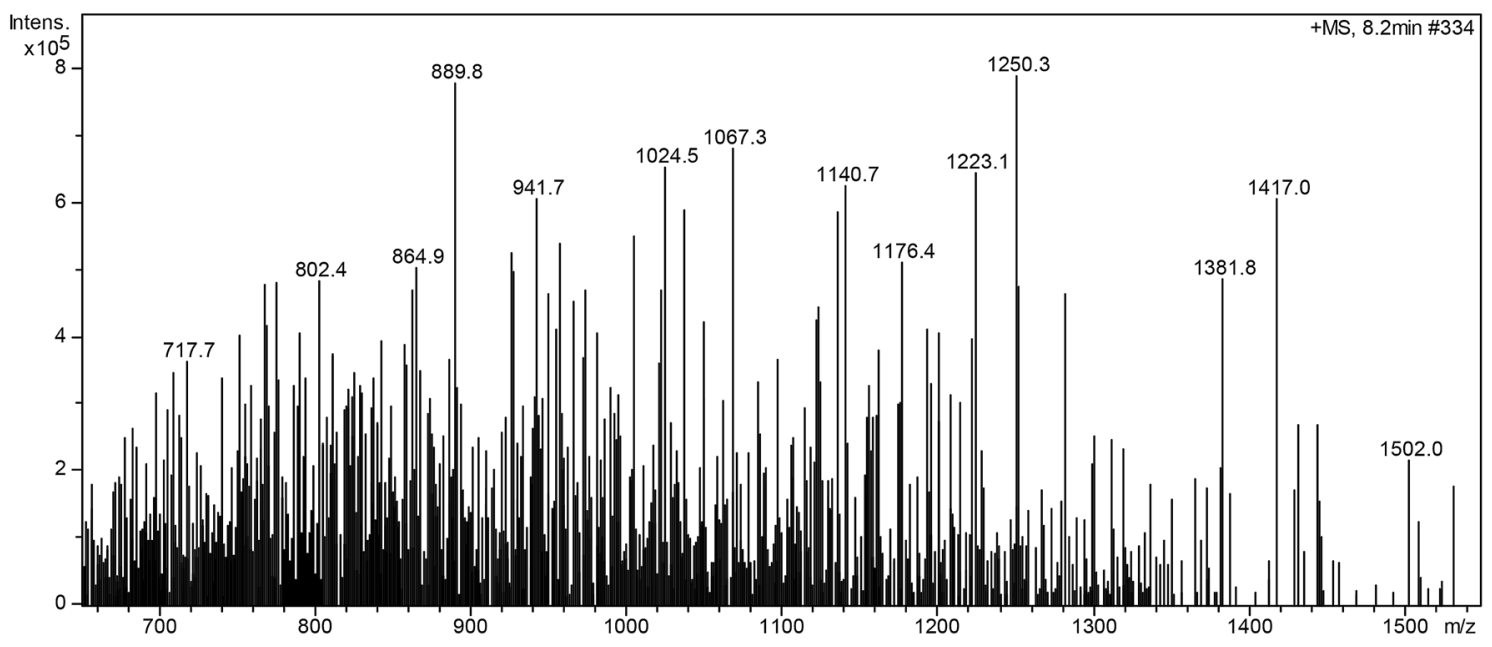

Figure 6. MS figure of the non-silicon oxygen bleaching stabilizer.

in single factor test, $\mathrm{D}_{2}$ was selected. Factor $\mathrm{E}$ was the fourth major factor. For the chelated iron value, $\mathrm{E}_{1}$ was the best, the reaction time was $4 \mathrm{~h}$, and for chelated calcium value, $\mathrm{E}_{2}$ was the best $(4.5 \mathrm{~h})$. Because of the more important influenced the chelated iron value than the chelated calcium value on this experiment, and the reason of energy saving, $E_{1}$ was selected. Factor $C$ was the secondary influencing factor. For the chelated iron value, $C_{3}$ was the best, and for the chelated calcium value, $\mathrm{C}_{4}$ was the best, because factor $\mathrm{C}$ had very little affect on both indicators, so $\mathrm{C}_{3}$ was selected to save the cost. So the final synthetic process of non-silicon oxygen bleaching stabilizer was $\mathrm{A}_{3} \mathrm{~B}_{4} \mathrm{C}_{3} \mathrm{D}_{2} \mathrm{E}_{1}$, that was, the dosage of magnesium sulfate $2 \mathrm{~g}$ (the mass ratio of acrylic acid and magnesium sulfate was $5: 1$ ), the concentration of acrylic monomer $25 \%$, the dosage of initiator $5 \%$ of the mass of acrylic monomer, the reaction temperature was at $65^{\circ} \mathrm{C}$ and the total reaction time was $4 \mathrm{~h}$.

The optimized process determined by orthogonal experiment was used for verification experiments, and the properties of the product were determined. The results showed that the chelated iron value was as high as $239.314 \mathrm{mg} / \mathrm{g}$, and the chelated calcium value was as high as $145.000 \mathrm{mg} / \mathrm{g}$, which was much higher than any one of the results on single factor and orthogonal experiments, so the synthesis process of the non-silicon oxygen bleaching stabilizer was feasible.

Mass spectra analysis. To determine the molecular weight of the product and its distribution, the mass spectrometer of 6310 Ion Trap LC-MS by Agilent Corporation (USA) was used. As shown in Fig. 6, the molecular weight of the product was distributed in the range of 700-1500, the main range was in $890-1250$. The figure showed that the highest peaks were at 889.8 and 1250.3, it indicated that the molecular weight of the product was mainly distributed in this range, and there were more strong peaks within this range, such as $941.7,1024.5$, $1067.3,1140.7,1223.1$, etc., indicated the molecular weight distribution was relatively uniform, ranging from 890 to 1250 .

Infrared spectra analysis. Spectrum One Fourier Transform Infrared Spectrometer by PerkinElmer (USA) was used. The infrared spectrum of synthesized non-silicon oxygen bleaching stabilizer was shown in Fig. 7.

Bands at around $1700 \mathrm{~cm}^{-1}$ were assigned to poly(acrylic acid). In Fig. 7, the peak observed at $1713.29 \mathrm{~cm}^{-1}$ was due to $\mathrm{C}=\mathrm{O}$ stretching vibration in poly(acrylic acid), at $1163.38 \mathrm{~cm}^{-1}$ was due to $\mathrm{C}-\mathrm{O}$ stretching vibration in poly(acrylic acid). The strong band was found at around $1710 \mathrm{~cm}^{-1}$ and a characteristic band was found at $1450 \mathrm{~cm}^{-1}$, it indicated that polyketones was producd. The narrow absorption peak observed at $1435.39 \mathrm{~cm}^{-1}$ was assigned to the presence of a trace polyketone. A broad absorption peak observed at $3402.94 \mathrm{~cm}^{-1}$ indicated that hydrogen bonds were formed between the molecules in the product, including the formation of hydrogen bonds between molecules, two-molecular associations, or multimolecular associations ${ }^{34}$. The weaker absorption peaks observed at 2966.78 and $2642.06 \mathrm{~cm}^{-1}$ indicated that $\mathrm{O}-\mathrm{H}$ and other groups in the molecule formed hydrogen bonds, conduciving to form chelating bonds for increasing the chelating performance of the product. In addition, the absorption peaks observed at 797.64 and $605.67 \mathrm{~cm}^{-1}$ indicated that the product contained substituted benzene, unsaturated double bonds, which may be the reason that the product contained less unreacted acrylic monomer. Infrared spectrum showed that the target product was obtained.

Determination of the synthesized stabilizer dosage in bleaching linen yarn. Effect of the stabilizer dosage on decomposition rate of $\mathrm{H}_{2} \mathrm{O}_{2}$. In hydrogen peroxide bleaching, stabilizer must be added to inhibit the excessive decomposition of hydrogen peroxide and avoid severe damage to the yarn or fabric. And the dosages of the stabilizer may affect the decomposition rate of hydrogen peroxide. The bleaching process was carried out with a bath ratio of 1:20. Each bleaching solution contained $\mathrm{H}_{2} \mathrm{O}_{2} 5 \mathrm{~g} / \mathrm{L}, \mathrm{Na}_{2} \mathrm{CO}_{3} 2.5 \mathrm{~g} / \mathrm{L}, \mathrm{NaOH} 1 \mathrm{~g} / \mathrm{L}$, wetting agent JFC $1 \mathrm{~g} / \mathrm{L}$, stablizer $(2,4,6,8 \mathrm{~g} / \mathrm{L})$, bleaching at $90-95^{\circ} \mathrm{C}$ for $60 \mathrm{~min}$. The linen yarn was $10 \mathrm{~g}$ for each experiment. The decomposition rate of hydrogen peroxide under different stabilizer dosages was measured 


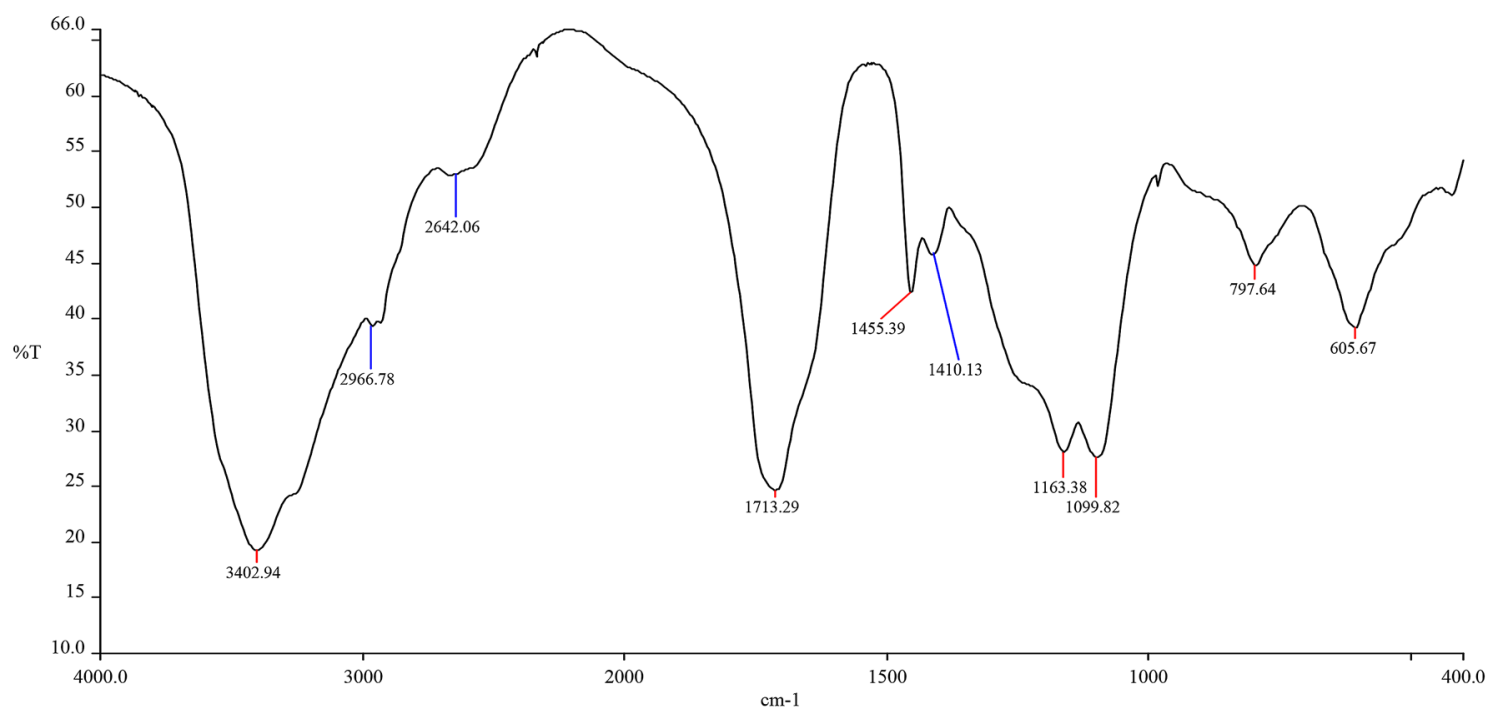

Figure 7. IR Figure.

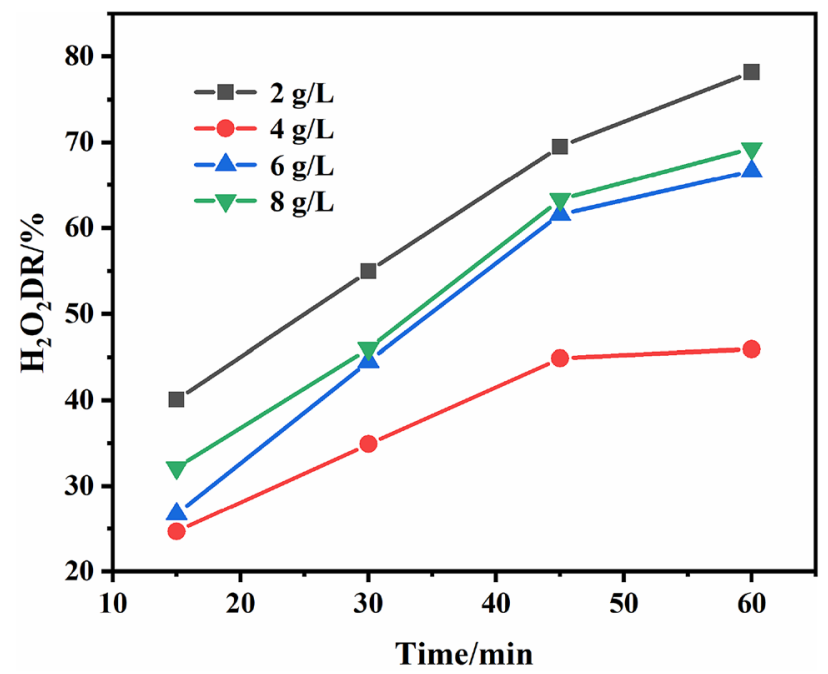

Figure 8. Effect of the synthesized stabilizer on $\mathrm{H}_{2} \mathrm{O}_{2}$ decomposition rate.

every $15 \mathrm{~min}$. The average value was taken from the two bleaching tests. The effect of the synthesized stabilizer on $\mathrm{H}_{2} \mathrm{O}_{2}$ decomposition rate is shown in Fig. 8.

From the results shown in Fig. 8, when 2 g/L synthesized stabilizer were added in the bleaching bath, the decomposition rate of $\mathrm{H}_{2} \mathrm{O}_{2}$ was the highest at any time, it was closed to $80 \%$ at bleaching $60 \mathrm{~min}$. The reason was that the stabilizer dosage was too small to inhibit the decomposition of $\mathrm{H}_{2} \mathrm{O}_{2}$. When the stabilizer was added to $4 \mathrm{~g} / \mathrm{L}$, the decomposition rate of $\mathrm{H}_{2} \mathrm{O}_{2}$ decreased significantly by as much as $20 \%$. The decomposition rate of $\mathrm{H}_{2} \mathrm{O}_{2}$ was $24.67 \%$ at bleaching $15 \mathrm{~min}$, and did not exceed $50 \%$ at the end of bleaching $60 \mathrm{~min}$. At this time, the stabilizer dosage was relatively moderate. The stabilizer dosage in bleaching bath was not high, and the polymer chains can be diastolic, which can adsorb and chelate the catalytic material well, and effectively reduced the ineffective decomposition of $\mathrm{H}_{2} \mathrm{O}_{2}$. While it increased to $6 \mathrm{~g} / \mathrm{L}$, the decomposition rate of $\mathrm{H}_{2} \mathrm{O}_{2}$ did not continue to reduce. The stabilizer were added to $8 \mathrm{~g} / \mathrm{L}$, the decomposition rate was not continuing to reduce. It seems that increasing the amount of stabilizer can no longer reduce the decomposition rate of $\mathrm{H}_{2} \mathrm{O}_{2}$. With increased the stabilizer dosage, the polymer chain segments can not stretch freely, and the probability of mutual adsorption and chelation between the curled polymer chains increased, which weakened the adsorption and chelation of the surrounding substances catalyzing the decomposition of $\mathrm{H}_{2} \mathrm{O}_{2}$, and the ineffective decomposition of $\mathrm{H}_{2} \mathrm{O}_{2}$ increased. So the suitable dosages of this stabilizer in the bleaching of linen yarn should be $4 \mathrm{~g} / \mathrm{L}$.

Effect of stabilizer dosages on whiteness. Different dosages of the stabilizer leaded to different inhibition on the decomposition of $\mathrm{H}_{2} \mathrm{O}_{2}$, which directly affects the effective decomposition or excessive decomposition of $\mathrm{H}_{2} \mathrm{O}_{2}$, and affects the whiteness of the bleached textile materials. Invalid or excessive decomposition of $\mathrm{H}_{2} \mathrm{O}_{2}$ not only 


\begin{tabular}{|l|l|l|l|l|}
\hline Dosages of the synthesized stabilizer $/(\mathbf{g} / \mathbf{L})$ & $\mathbf{2}$ & $\mathbf{4}$ & $\mathbf{6}$ & $\mathbf{8}$ \\
\hline Whiteness/\% & $64.30 \pm 0.15$ & $66.81 \pm 0.58$ & $65.50 \pm 0.39$ & $61.86 \pm 0.51$ \\
\hline
\end{tabular}

Table 3. The effect of synthesized stabilizer dosage on whiteness.
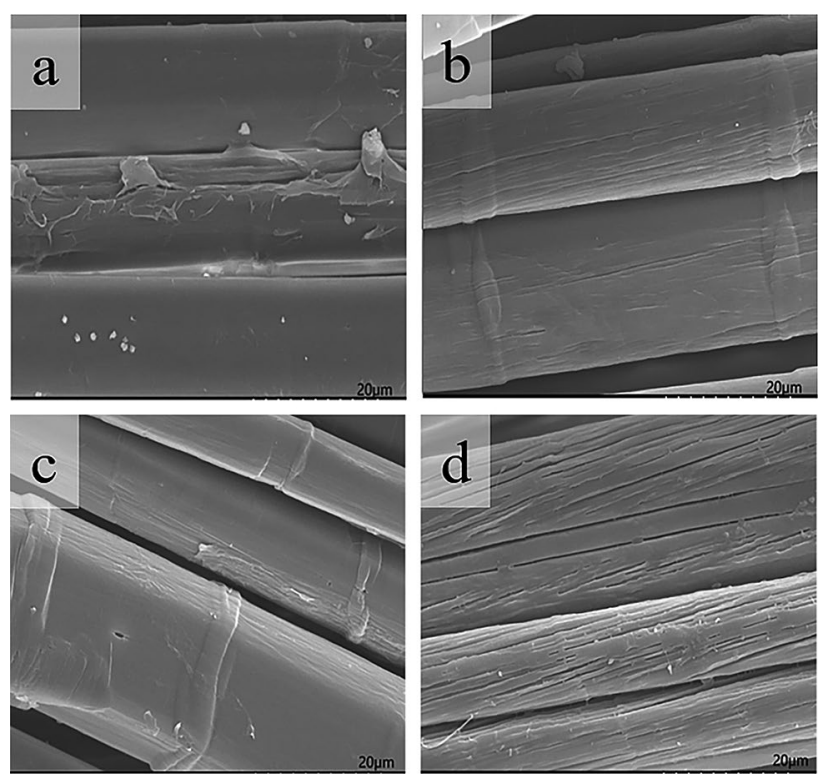

Figure 9. SEM photos of the bleached samples with different dosages of the synthesized OBS. (a) 2 g/L OBS; (b) $4 \mathrm{~g} / \mathrm{L} \mathrm{OBS}$; (c) $6 \mathrm{~g} / \mathrm{L} \mathrm{OBS;} \mathrm{(d)} 8 \mathrm{~g} / \mathrm{L}$ OBS.

affects the whiteness, but also causes fiber demage. The effect of synthesized stabilizer dosage on whiteness was shown in Table 3.

From the results shown in Table 3, when the bleaching bath contained $4 \mathrm{~g} / \mathrm{L}$ synthesized OBS, the whiteness of the bleached sample reached the maximum $66.81 \%$. Continued to increase the stabilizer dosage, the whiteness decreased instead. When the stabilizer dosages reached $8 \mathrm{~g} / \mathrm{L}$, the whiteness of the bleached sample was down to $61.86 \%$. Because of exceeding $4 \mathrm{~g} / \mathrm{L}$ of the stalizer dosage, the decomposition rate of $\mathrm{H}_{2} \mathrm{O}_{2}$ increased, resulting in ineffective decomposition, so the whiteness also decreased. Therefore, the most suitable amount of this stabilizer in the bleaching of linen yarn was $4 \mathrm{~g} / \mathrm{L}$.

Surface examination by scanning electron microscopy (SEM). SEM examination of the linen yarn was made with S-3400 Scanning Electron Microscope by Hitachi Co. (Japan). SEM photos of the bleached samples with different dosages of the synthesized OBS were given in Fig. 9.

It can be seen from Fig. 9, the slight change on the fiber surface was clearly observed. While the stabilizer dosage was $2 \mathrm{~g} / \mathrm{L}$ (a) , there were granular solids on the fiber surface, which may be the colloidal material existed in the flax fiber itself, or metal ions and impurities chelated by the stabilizer adhesived to the fiber, resulted in poor handle and lower whiteness. While OBS dosage was $4 \mathrm{~g} / \mathrm{L}$ (b), there were no solid particles on the fiber surface, and the surface was smooth and loose, so it had good handle and higher whiteness, which helped to subsequent dyeing process. While OBS dosage was $6 \mathrm{~g} / \mathrm{L}$ (c), there was basically no solid substance attached on the fiber surface, and the surface was relatively smooth and loose, with better handle and higher whiteness, but appeared some clear micropores. When OBS dosage was $8 \mathrm{~g} / \mathrm{L}(\mathrm{d})$, there were less amount of solid particles on the surface of the fiber, and appeared severe cracks on the fiber surface, which maybe inevitably decrease the fiber breaking tenacity.

According to the influences of the above-mentioned dosages of the synthesized OBS on the decomposition rate of $\mathrm{H}_{2} \mathrm{O}_{2}$ and whiteness of the bleached sample, in combination with the analysis of SEM photos, The dosages of the synthesized OBS should be $4 \mathrm{~g} / \mathrm{L}$.

\section{Conclusions}

In this study, a non-silicon oxygen bleaching stabilizer, based on acrylic acid and magnesium sulfate, was synthesized for hydrogen peroxide bleaching. The optimal synthesizing process of the stablizer was determined, namely, acrylic acid monomer concentration $25 \%$, initiator potassium persulfate $5 \%$ of the mass of acrylic monomer, reacted at $65^{\circ} \mathrm{C}$ for $4 \mathrm{~h}$, then added magnesium sulfate (the mass ratio of acrylic acid and magnesium sulfate is 5:1), continued to react for $1 \mathrm{~h}$. The synthesized complex compound of poly(acrylic acid) and magnesium salt had good chelating effect, the chelated iron value reached $239.314 \mathrm{mg} / \mathrm{g}$, and the chelated calcium value reached 
$145 \mathrm{mg} / \mathrm{g}$, so it can effectively inhibit the rapid decomposition of hydrogen peroxide caused by the presence of metal ions in the bleaching solution. The molecular weight of the polymer was uniformly distributed, ranging from 890 to 1250 . The bleaching results indicated that the optimal dosage applied to linen yarn was $4 \mathrm{~g} / \mathrm{L}$. the decomposition rate of hydrogen peroxide was no more than $50 \%$ in $60 \mathrm{~min}$, the whiteness reached $66.81 \%$. Compared with sodium silicate as the traditional oxygen bleaching stabilizer, the synthesized poly(acrylic acid) magnesium salts complex compound had the similar whiteness performence, and the bleached samples had much better handle and no silicon scale generated, it can effectively solve the silicon scale problem.

This study is expected to provide contribution to the future develop of non-silicon oxygen bleaching stabilizer. For future work, the synthesized poly(acrylic acid) magnesium salts complex compound will be applied for banana and hemp fibers bleaching and the related environmental friendly bleaching processes will be investigated.

Received: 17 February 2021; Accepted: 4 May 2021

Published online: 14 May 2021

\section{References}

1. Wuorimaa, A., Jokela, R. \& Aksela, R. Recent developments in the stabilization of hydrogen peroxide bleaching of pulps: An overview. Nord. Pulp Pap. Res. J. 21(04), 435-443 (2009).

2. An, D. et al. Preparation, characterization, and application of high-whiteness agar bleached with hydrogen peroxide. Food Hydrocolloids 113, $106520(2021)$.

3. He, H., Lu, S. \& Song, M. Research on the bleaching process of the non-silicon oxygen bleaching stabilizer. Appl. Mech. Mater. 685, 64-67 (2014).

4. Yu, D., Wu, M. \& Lin, J. Establishment of an effective activated peroxide system for low-temperature cotton bleaching using synthesized tetramido macrocyclic iron complex. Fibers and Polymers. 18(9), 1741-1748 (2017).

5. Li, M., Yin, J. \& Hu, L. Effect of hydrogen peroxide bleaching on anionic groups and structures of sulfonated chemo-mechanical pulp fibers. Colloids Surf., A 585, 1-36 (2020).

6. Hu, C., Lv, T. \& Zhang, Y. Study on synthesis and characteristics of macromolecule chelator. J. Appl. Polym. Sci. 36(2), 158-160 (2007).

7. Wang, B. et al. Summary of development on stabilizer in $\mathrm{H}_{2} \mathrm{O}_{2}$-bleaching. Heilongjiang Text. 153(3), 4-6 (2020).

8. Ohmura, S., Yokoyama, T. \& Matsumoto, Y. Significance of benzylic hydroxymethylene group in the reaction of lignin side-chain with active oxygen species under oxygen bleaching conditions. J. Wood Sci. 59(4), 337-343 (2013).

9. Xu, C., Hinks, D. \& Shamey, R. Bleaching cellulosic fibers via pre-sorption of N-[4-(triethylammoniomethyl)- benzoyl]-butyrolactam chloride. Cellulose 17(4), 849-857 (2010).

10. Sharnina, L. V., Vladimirtseva, E. L. \& Odintsova, O. I. Hydrogen peroxide as a polymerization initiator for silicate bleaching solution stabilizers. Russ. J. Gen. Chem. 89(3), 580-585 (2019).

11. Fu, D. et al. Preparation and property analysis of polyacrylate dispersant for calcium carbonate. Colloids Surf. A 326(3), 122-128 (2008).

12. Topalovic, T., Nierstrasz, V. A. \& Warmoeskerken, M. Model system for mechanistic study of catalytic bleaching of cotton. Fibers Polym. 11(1), 72-78 (2008).

13. Ohmura, S., Yokoyama, T. \& Matsumoto, Y. Significance of benzylic hydroxymethylene group in the reaction of lignin side-chain with active oxygen species under oxygen bleaching conditions. J. Wood Sci. 59(4), 337-343 (2008).

14. Heli, K., Anna, S. \& Markku, H. Characterization of functional properties of poly in mechanical pulp bleaching conditions. Nord. Pulp Pap. Res. J. 24(1), 12-18 (2009).

15. Zhang, J. New development and application of energy-saving and emission reduction type pretreatment agents. Text. Aux. 31(1), 1-8 (2008)

16. Chen, R. Synthesis and application of polyphosphonate type oxygen bleaching stabilizer. Print. Dye. Aux. 14(5), 5-11 (1997).

17. Chen, R. Development of organic chelating agent and environment friendly varieties. Text. Dye. Finish. J. 28(2), 1-9 (2011).

18. Zhu, W., Gu, D. \& Wu, C. Preparation and application of non-silicon and hydrogen peroxide stabilizer NC-604. Dye. Finish. 6, 8-10 (2003).

19. Zhou, L. \& Luo, K. Preparation and application of high efficiency oxygen bleaching non-silicon stabilizer. Print. Dye. 6, 11-13 (2003).

20. Qi, W. \& Huang, W. Development and application of oxygen bleaching stabilizer FK-601. Shanghai Silks. 3, 28-30 (2008).

21. Florit, F. et al. Solution polymerization of acrylic acid initiated by redox couple Na-PS/Na-MBS: kinetic model and transition to continuous process. Processes 8(7), 1-19 (2008).

22. Shao, J. et al. Cold pad-batch bleaching of cotton fabrics with a TAED/ $\mathrm{H}_{2} \mathrm{O}_{2}$ activating system. Color. Technol. 126(2), 103-108 (2008).

23. Qin, X. et al. Low-temperature bleaching of cotton fabric with a binuclear manganese complex of 1,4,7-trimethyl- 1,4,7-triazacyclononane as catalyst for hydrogen peroxide. Colorat. Technol. 128(5), 410-415 (2012).

24. Taboada, E., Cabrera, G. \& Cardenas, G. Synthesis and charaeterization of new arylamine chitosan derivatives. J. Appl. Polym. Sci. 91(2), 807-812 (2004).

25. Luo, X. et al. Whitening citric acid treated cotton fabrics by a TBCC-activated peroxide post-bleaching. Cellulose 27, 5367-5376 (2020).

26. Li, L. \& Li, Q. Combined scouring and bleaching of cotton/linenblends by a near-neutral activated peroxide system. Fibres Text. Eastern Eur. 28(6), 104-109 (2020).

27. Wang, X. \& Tang, J. An evaluation of the effectiveness of applying a gelatin-copper complex in the low-temperature bleaching of cotton with hydrogen peroxide. Color. Technol. 133(4), 300-304 (2017).

28. Liu, J. Effect of hydrogen peroxide decompostion rate on the performance of bleached cotton fabrics in hydrogen peroxide bleaching system. Text. Dye. Finish. J. 41 (01), 23-26+53 (2019).

29. Yin, C. et al. Low-temperature bleaching of cotton fabric using a copper-based catalyst for hydrogen peroxide. Color. Technol. 131(1), 66-71 (2015).

30. Liu, K., Zhang, X. \& Yan, K. Bleaching of cotton fabric with tetraacetylhydrazine as bleach activator for $\mathrm{H}_{2} \mathrm{O}_{2}$. Carbohydr. Polym. Sci. Technol. Asp. Ind. Important Polysaccharides 188, 221-227 (2018).

31. Topalovic, T., Nierstrasz, V. A. \& Warmoeskerken, M. Model system for mechanistic study of catalytic bleaching of cotton. Fibers Polym. 11(1), 72-78 (2010).

32. Luo, X. et al. An eco-friendly way to whiten yellowish anti-wrinkle cotton fabrics using TBCC-activated peroxide low-temperature post-bleaching. Cellulose 26, 3575-3588 (2019).

33. Meena, B. I. \& Kaizer, J. Design and fine-tuning redox potentials of manganese(II) complexes with isoindoline-based ligands: $\mathrm{H}_{2} \mathrm{O}_{2}$ oxidation and oxidative bleaching performance in aqueous solution. Catalysts 10(4), 404 (2020). 
34. Altay, P. et al. Facile synthesis of a novel, highly effective, more sustainable and cost-effective cationic bleach activator for cotton: $\mathrm{N}-[4-(\mathrm{N}, \mathrm{N}, \mathrm{N})$-triethylammoniumchloride-butyryl] capro-lactam. Cellulose 26(4), 2849-2860 (2020).

35. Gupta, A. et al. Low temperature synthesis of NbC/C nano-composites as visible light photoactive catalyst. Sci Rep. 8, 13597. https://doi.org/10.1038/s41598-018-31989-z (2018).

\section{Acknowledgements}

This work was financially supported by the General items of basic scientific research and business expenses of provincial universities in Heilongjiang Province (135409313), Ministry of Education Cooperative Education Project of China (201802142010).

\section{Author contributions}

J.L. and C.L. wrote the main manuscript text. All authors reviewed the manuscript.

\section{Competing interests}

The authors declare no competing interests.

\section{Additional information}

Correspondence and requests for materials should be addressed to C.L.

Reprints and permissions information is available at www.nature.com/reprints.

Publisher's note Springer Nature remains neutral with regard to jurisdictional claims in published maps and institutional affiliations.

(c) (i) Open Access This article is licensed under a Creative Commons Attribution 4.0 International License, which permits use, sharing, adaptation, distribution and reproduction in any medium or format, as long as you give appropriate credit to the original author(s) and the source, provide a link to the Creative Commons licence, and indicate if changes were made. The images or other third party material in this article are included in the article's Creative Commons licence, unless indicated otherwise in a credit line to the material. If material is not included in the article's Creative Commons licence and your intended use is not permitted by statutory regulation or exceeds the permitted use, you will need to obtain permission directly from the copyright holder. To view a copy of this licence, visit http://creativecommons.org/licenses/by/4.0/.

(C) The Author(s) 2021 